\title{
loT-Based System Monitoring of the Sleep Environment - A Study Aimed at the Elderly
}

\author{
Leandro Y. Mano ${ }^{1,2,{ }^{*}}$, Igor G. Oliveira ${ }^{3}$, Luana O. Sawada ${ }^{1}$, Bruno S. Faiçal ${ }^{1}$ and \\ Jó Ueyama ${ }^{1}$
}

${ }^{1}$ Institute of Mathematical and Computer Sciences, University of São Paulo, São Carlos, São Paulo, Brazil

${ }^{2}$ Johann Bernoulli Institute for Mathematics and Computer Science, University of Groningen, Groningen, The Netherlands

${ }^{3}$ State University of North Paraná, Bandeirantes, Paraná, Brazil

\begin{abstract}
The aging process in our population can cause changes in people's sleeping patterns, more specifically in the elderly, by impairing their cognitive abilities, quality of life, and autonomy. Advances in Ubiquitous Computing and Internet of Things have contributed to the monitoring of such situations. In particular, the use of sensors to evaluate the environment and aspects related to the health and well-being of individuals, as well as providing event alerts. The main objective of this experiment is to propose a monitoring system based on both the responses of multiple sensors (brightness, microphone, accelerometer, and gyroscope) at runtime to classify the environment for elderly people's sleep quality. The results show that using embedded devices, and capturing environmental aspects through sensors, can develop solutions that offer more safety and comfort to the individuals' sleep quality environment.
\end{abstract}

Keywords: Ubiquitous Computing, Sensors, Multiple Sensors, Internet of Things, Quality of Sleep.

\section{INTRODUCTION}

Increasing life expectancy worldwide results in the growth of the elderly population in large urban centers, especially in developed countries [1-3]. Although much of the population is living longer, strategies are still needed to help elderly people with health and wellbeing, as they are more prone to accidents and chronic illnesses [2, 3]. In addition, they have difficulties or limitations in carrying out daily tasks, which can considerably affect their quality of life $[2,3]$. In this setting, technology can be used to enable elderly people to live more independently and safely using monitoring systems, assisting medical staff and family members in risk situations $[4,5]$.

Given the need to help individuals in health issues and the significant technological advance of recent times, the concept of Health "Smart" Homes has emerged [6], which combines concepts of Telemedicine, Internet of Things (loT) and Information Systems aiming to preserve remote health care in residential environments [1, 2, 7]. This approach has been widely explored in the literature [2, 4-9], proposing solutions that can assist in the monitoring of daily aspects, enabling greater independence and security for people with special needs, physical limitations and/or medical treatment $[2-5,9,10]$.

Address correspondence to this author at the Institute of Mathematical and Computer Sciences, University of São Paulo, São Carlos, São Paulo, Brazil; Tel: +55163373-9700; Fax: +55163373-8888;

E-mail: leandroyukiomano@gmail.com
Considering this, using multi-sensor devices can provide user context information more accurately, which can identify situations through contextual processing such as physical activity, heartbeat, fall occurrence, sleep quality, among other aspects [11]. Thus, science of a situation is the ability of a computer system to gain a comprehensive view and high level of abstraction of the application's contexts of interest that can be used in the decision-making process [11].

According to Ancoli-Israel et al.(1997) [12], some specific characteristics regarding monitoring the elderly are related to resting issues, namely: (i) reports of the elderly claiming to spend more time in bed due to advancing age; (ii) frequent nocturnal awakenings; (iii) there is a significant increase in hypersomnia complaints, which are often secondary to other diseases. Furthermore, elderly people have sleeprelated complaints resulting from specific physiological changes in the aging process or from diseases that can cause secondary sleep disorders [13]. The experience of unsatisfactory or insufficient sleep is very unpleasant and has a reflection on performance, behavior and well-being during activities of daily living [14].

Thus, this article uses multiple sensors to monitor the environmental conditions in which elderly people rest, which may provide clues about the aspects of the environment that influence unsatisfactory rest situations. This information monitored by this system will be used in future studies to compose a metric related to elderly individuals' sleep quality. It is 
important to mention that this study consists of the hypothesis of the possibility of monitoring aspects of an environment that relate to the quality of the resting environment.

Thus, the proposed system serves as the information base to provide environments with sufficient quality for elderly people to have a satisfactory rest [14]. Finally, it is important to highlight that the proposal has the characteristic of performing a non-intrusive monitoring, favoring elderly people's sense of independence.

The remainder of this paper is organized as follows: Section 2 deals with the application of health care monitoring in smart environments in the literature. Section 3 presents our proposal for environmental monitoring for sleep quality, use of sensors and embedded devices. Section 4 presents the methodology adopted and, in turn, Section 5 presents the results of the experiments performed. Finally, Section 6 presents some concluding remarks and points out possible future directions for this study.

\section{RELATED STUDIES}

Research in the area of Ubiquitous Computing, loT, and eHealth/mHealth $[2-5,7,9,15,16]$ has generated a wide range of methods that can extract patterns from the environment and/or user and use them to monitor and classify aspects related to the individual's health and well-being.

Chung and Oh (2006) [16] present an example of environmental monitoring and follow-up. The study presents a module to monitor the air quality of the home environment and makes use of humidity, temperature, $\mathrm{CO} 2$ and dust sensors, among others, added to the home system which includes surveillance camera and power switches to control lighting and power consumption to improve the environment monitoring function [16]. However, this approach differs from the proposed work in terms of aspects related to the individual, more precisely to the sleep quality environment, addressing only the issues of the home environment.

In the context of monitoring individual conditions, [17] propose the Home Health Care System (HHCS), based on Pervasive Computing for data acquisition from physiological and motion sensors to identify the patient's situation [17]. On the other hand, [4] propose an individualized residential monitoring loT architecture to monitor the patient's emotional state based on Machine Learning techniques. Thus, the system identifies the patient and classifies their emotional state by facial expressions and can issue alerts regarding the patient's emotional state under home treatment [4]. However, both approaches to home monitoring become totally or partially intrusive to the user, as they depend on the constant interaction with the devices, either through the sensors attached to their body or interaction for image acquisition, different from the proposed approach, based on the embedded device sensors for completely non-intrusive data acquisition. Moreover, both approaches do not relate the sleep quality environment, which may influence the treatment, improvement and daily life of individuals, especially the elderly population.

In turn, specifically referring to monitoring an individual's rest, [18] describe procedures, recommendations, findings and values for diagnoses used in sleep disorders. Polysomnography and multiple sleep latency testing are considered to be "gold standard" methods in most sleep disorders and narcolepsy. Specific questionnaires including sleep quality assessment, hypersomnia, sleep-disordered breathing, and sleep-wake rhythm are generally used to screen sleep disorders and studies on sleep [18]. On the other hand, [15] developed a platform to unify SleepSmart (a multisensor mattress pad to detect body positions, body temperature, and heart and breathing rate) and Morpheus (a mattress actuation system to encourage people to roll over in bed to alleviate snoring and apnea) for a more uniform system of monitoring and improving sleep quality [15]. However, although they address sleep-related issues, both approaches require user interaction with devices, either by completing questionnaires or by body interaction, different from this work proposal that monitors the user's sleep environment non-intrusively.

Table 1 presents a synthesis of all the researches found in the literature and detailed above. Studies were analyzed considering the following features: (i) environment monitoring; (ii) non-intrusive interactions and; (iii) classification of the environment's quality for sleep.

A significant increase has recently been observed in studies that address the context that the system is proposed, Health Smart Homes [1, 3, 4, 18]. These studies contribute with techniques to develop solutions capable of identifying, through sensors and/or information, a certain aspect of the user. 
Table 1: Synthesis of Researches Found in the Literature

\begin{tabular}{|c|c|c|c|}
\hline Related studies & Environment monitoring & Non-intrusive interaction & Quality for sleep \\
\hline Chung and Oh (2006) & $\sqrt{ }$ & $\times$ & $\sqrt{ }$ \\
\hline Mano et al. (2016) & $\times$ & $\sqrt{ }$ & $\times$ \\
\hline Carvalho et al. (2010) & $\times$ & $\times$ & $\sqrt{ }$ \\
\hline Togeiro and Smith (2005) & $\times$ & $\times$ & $\sqrt{ }$ \\
\hline Van Der Loos et al. (2003) & $\sqrt{ }$ & $\sqrt{ }$ \\
\hline Proposal & $\sqrt{ }$ & & $\times$ \\
\hline
\end{tabular}

It should be mentioned, however, that there is a lack of studies in the area of Health Smart Homes that address concepts related to Ubiquitous Computing, as well as issues related to identifying the quality of the users' resting environment, as the environment is considered an important factor when analyzing sleep quality as it can directly affect the quality of life and elderly users' behaviour [12, 14]. These characteristics are met in this work by collecting and analyzing data from mobile device sensors non-intrusively. This proposal allows computer systems to use environmental quality information as a complementary feature in the user's sleep analysis.

\section{SLEEP QUALITY ENVIRONMENT MONITORING SYSTEM}

Coupled with the growth of computer system users, as well as easy access to telecommunications, is the possibility to capture and analyze data generated by individuals' behavior in different contexts $[2,4,5,9$, 10]. Therefore, sleep quality detection can be used to develop new ways of user interaction and, later, to move towards cognitive devices [19]. Information technologies that can interact and help in daily life are one of the ways to meet the preferences and needs of the elderly for a better quality of life [2].

Thus, based on research $[20,21]$ that attempts to understand factors that positively or negatively affect individuals' sleep quality the following can be highlighted: intense lighting, occurrence of noise and movement during the night. Thus, the basic information collected by the proposed system is: (i) brightness, (ii) sound intensity and (iii) device motion.

The current proposal allows for non-intrusive monitoring through mobile devices and their respective sensors, allowing new devices to be integrated to collect different information to achieve a higher rate of assertive decisions. Figure 1 illustrates the logical layers of the proposed system for environmental monitoring and the respective basic information collected. In layer 1, environmental data are acquired and transmitted. Later, in layer 2 , the characteristics are processed to identify the quality of the resting environment.

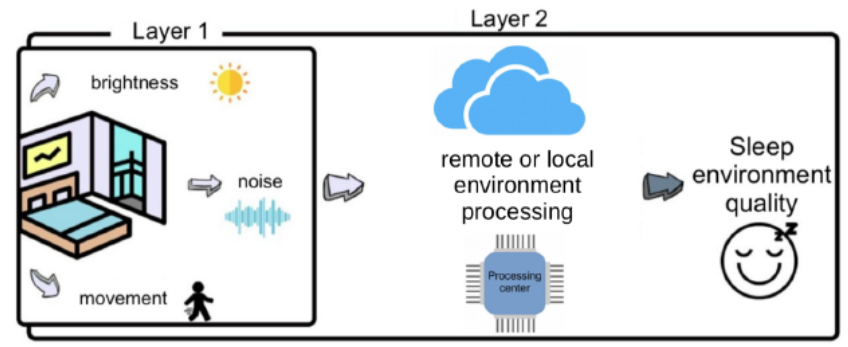

Figure 1: Non-intrusive scenario to environment monitoring for sleep quality.

Non-intrusive monitoring performed by the system refers to the transparent behavior to the user, who does not understand how the system works. In addition, the system only performs an action (e.g., communicates to family members and/or medical staff about a long period of insomnia) when an inappropriate situation is detected. Inadequate situations can be understood as those that present specific characteristics or those that do not consider elderly people's standard behavior, as alert situations that need to be reported.

Given the nature of the proposal, the requirements of this system are data consistency and high service availability. System consistency is related to stable behavior and valid real environment values. High availability is necessary because it is a monitoring system and if the system becomes unavailable it will not be able to identify inadequate situations during this period.

As previously described, the basic operation of the monitoring system collects three pieces of environmental information, namely: (i) brightness, (ii) sound intensity (noise) in the environment and (iii) 
device motion. This information can be explored individually and/or combined due to the ease of representing the context of the environment. The following are the sensors used for environmental monitoring to classify sleep quality:

- Brightness: brightness sensor that captures the presence of reflected light in the device. From this information, the presence or absence of light and its intensity can be determined;

- Sound amplitude: Noise sensor (microphone) that captures the presence of sound in the environment. It worth mentioning that for this study no data regarding the meaning of the sound were captured, maintaining the user's privacy. the sound amplitude present in the environment can be determined from this value;

- Accelerometer: Motion sensor that captures the variation in the acceleration that a body exerts in space in three-dimensional form, that is, on the $X, Y$ and $Z$ axes. The displacement of a moving body can be determined from these values, and;

- Gyroscope: motion sensor that captures the rotation change that a body exerts in space in three-dimensional form, that is, on the $X, Y$ and $Z$ axes. The rotation and change of direction of a body can be determined from these values.

\section{METHODOLOGY}

Thus, considering the data set obtained by four distinct sensors (brightness, microphone, accelerometer and gyroscope) in order to monitor and classify the environmental conditions, considering a pleasant and comfortable night for the elderly to rest, the classification of the environment is given considering the following labels: 1) unsatisfactory; 2) moderate and; 3) satisfactory.

The experiments presented were evaluated at two different times. The first evaluation focuses on the analysis of the sensitivity and quality of the data provided by the sensors, i.e., how much each sensor selected is sensitive to changes in the environment and whether such changes are noticeable by the sensors. The second evaluation verified the consistency of the system in the monitoring for a continuous period, where four different scenarios were established, as shown in Table 2.
Table 2: Experiment Scenarios for Environment Monitoring

\begin{tabular}{|c|c|c|c|}
\hline Scenario & Brightness & Noise & Movement \\
\hline \hline 1 & Not present & Not present & Moderate \\
\hline 2 & With brightness & Not present & Moderate \\
\hline 3 & Not present & With noise & Moderate \\
\hline 4 & With brightness & With noise & Moderate \\
\hline
\end{tabular}

Table 2 shows the environments submitted to the proposed system monitoring, namely: 1) totally dark environment and no noise present; 2) ambient with lighting and no noise; 3) environment without lighting and with noise and; 4) ambient with lighting and presence of noise. Moreover, all environments presented, for a short time, some kind of movement, to show access to the bathroom, intense nocturnal movement or even insomnia.

The environment used for the experiments was a room in a home and to vary the aspects in the environment the following were used: for the lighting, the bedroom ceiling light and the lighting provided by a video device (notebook); to generate noise, a sound device (sound system) that emitted constant or interleaved sounds was used; for the movement of the monitoring device, the user performed intentional movements. Moreover, to provide sufficient detail for possible replication of the experiments, the device used was the Samsung Galaxy S6 smartphone, Android OS v5.0.2, Octa-core $(4 \times 2.1 \mathrm{GHz}$ Cortex-A57 \& 4x1.5 GHz Cortex-A53).

The analyses and discussions of the experiments performed will be presented next.

\section{RESULTS AND DISCUSSION}

Initially, the tests were conducted to verify the sensitivity and quality of the data provided by the selected sensors. The results presented refer to the 60minute monitoring period, where the variance of the data collected on the $Y$ axis can be observed. Figure 2 presents the data distribution reported by the sensor regarding the brightness in two different environments: (i) without lighting and (ii) with lighting generated by the ambient lamp. In turn, Figure $\mathbf{3}$ shows the distribution of noise data, where (i) a quiet environment and (ii) an environment with constant noise generated by the audio device were monitored. Finally, Figure 4 shows the data distribution related to the motion of the 
identified device, where it was tested with the (i) fully immobile device and (ii) the user's movement. It is worth mentioning that to identify the movement, the data obtained by the accelerometer and gyroscope are combined to calculate the applied motion variance.

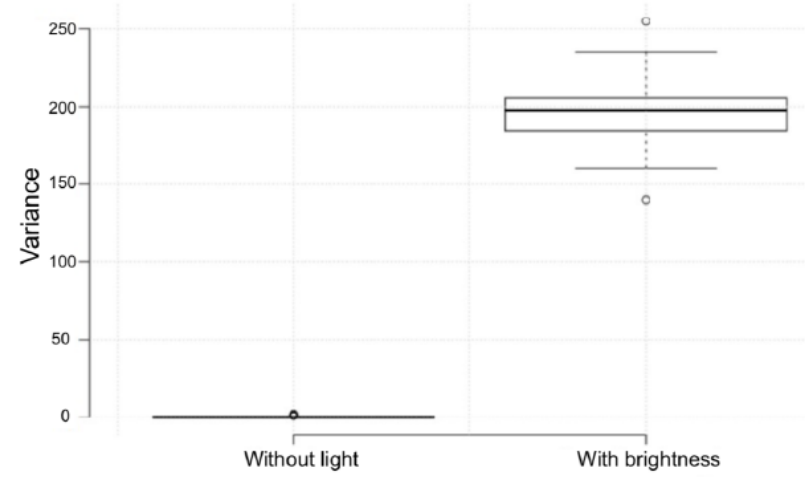

Figure 2: Boxplot showing the sensitivity and quality of the brightness sensor.

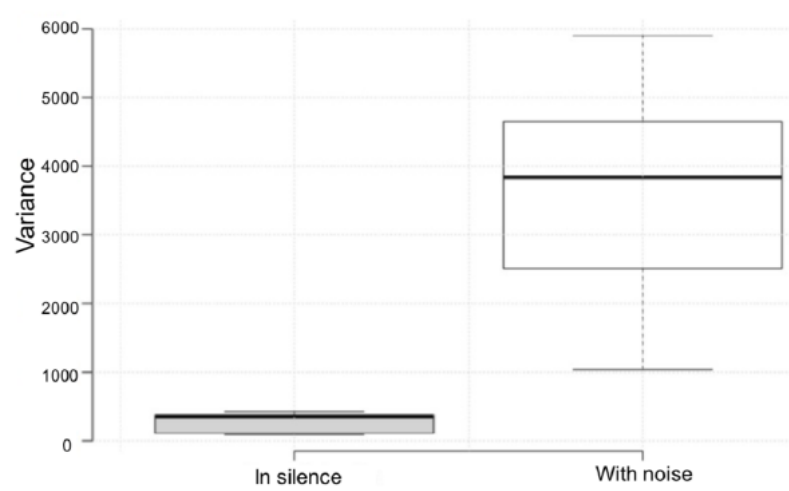

Figure 3: Boxplot showing the sensitivity and quality of the noise sensor (microphone).

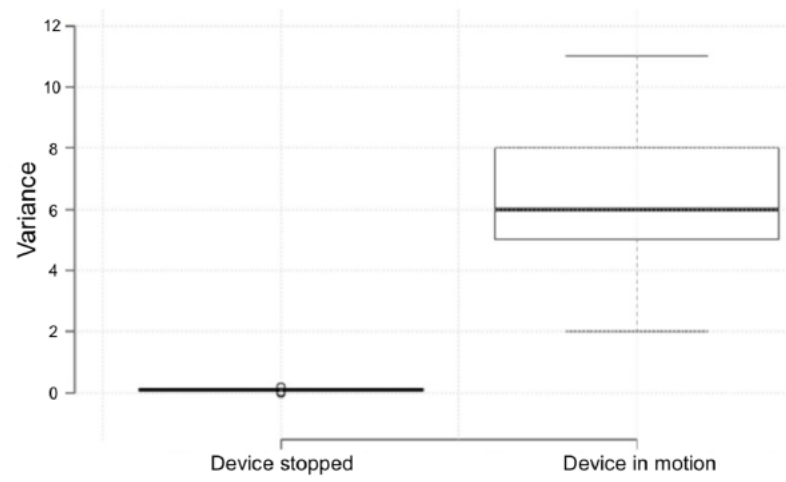

Figure 4: Boxplot showing the sensitivity and quality of the motion sensor (accelerometer and gyroscope.

These results demonstrate that the selected sensors present an acceptable sensitivity in order to identify the differences between the environments. The acceptable term refers to the fact that the data do not overlap, allowing a clear division between the data collected in each environment. Thus, it is believed that these results show that such information monitors the variation of a real environment during the night.

Based on these results and the labels to classify the environment at a given time, possibly at night, a score is defined for the ranges of values that reflect the characteristic of the environment in question. Table 3 presents, according to the sensitivity observed in the first evaluation, the range of values for the intervals of each characteristic (low, moderate and high) considered of the environment.

Table 3: Intensity Range

\begin{tabular}{|c|c|c|}
\hline Sensors & Range & Value \\
\hline \hline \multirow{3}{*}{ Brightness } & $0-80(\mathrm{l})$ & 0 \\
\cline { 2 - 3 } & $81-160(\mathrm{~m})$ & 1 \\
\cline { 2 - 3 } & $160-\ldots(\mathrm{h})$ & 2 \\
\hline \multirow{3}{*}{ Noise } & $0-1000(\mathrm{l})$ & 0 \\
\cline { 2 - 3 } & $1001-3000(\mathrm{~m})$ & 1 \\
\cline { 2 - 3 } & $3001-\ldots(\mathrm{h})$ & 2 \\
\hline \multirow{3}{*}{ Movement } & $0-2(\mathrm{l})$ & 0 \\
\cline { 2 - 3 } & $2.1-4(\mathrm{~m})$ & 1 \\
\cline { 2 - 3 } & $4.1-\ldots(\mathrm{h})$ & 2 \\
\hline
\end{tabular}

The ranges of values are used to classify the environment according to the following criteria: result of the sum of the values for each characteristic of the environment (defined in the column Value in Table 3) less than or equal to 1 classifies the environment as satisfactory for sleep; a result equal to 2 classified as the environment with moderate sleep conditions and; result of the sum equal or above 3 classifies the environment as unsatisfactory for sleep (see Table 4).

Table 4: Environment Classification for Test

\begin{tabular}{|c|c|}
\hline Result of the sum of values & Environment \\
\hline \hline defined in Table 3 & Classification \\
\hline$\leq 1$ & Satisfactory \\
$=2$ & Moderated \\
\hline$\geq 3$ & Unsatisfactory \\
\hline
\end{tabular}

For example, an environment with brightness 1, noise 0 and movement 0 is considered an environment 
satisfactory for sleeping, while an environment with brightness 1 , noise 0 and movement 2 is considered an unsatisfactory sleep environment. Regarding this, we considered the researches [20,21] that shows aspects related to intense lighting, noise generation and movement during the night concerning sleep quality.

In the second phase of the experiments, the system consistency analysis was carried out while monitoring for an extended period, where the scenarios presented in Table $\mathbf{2}$ were considered. Figure $\mathbf{5}$ presents the monitoring period of all scenarios, which had a duration of eight hours, and at each one-hour interval the environment was classified to verify the quality in terms of rest. In these experiments, the system proved to be consistent and highly available, as it constantly ran throughout the experiment and did not show inadequate values for environmental monitoring.

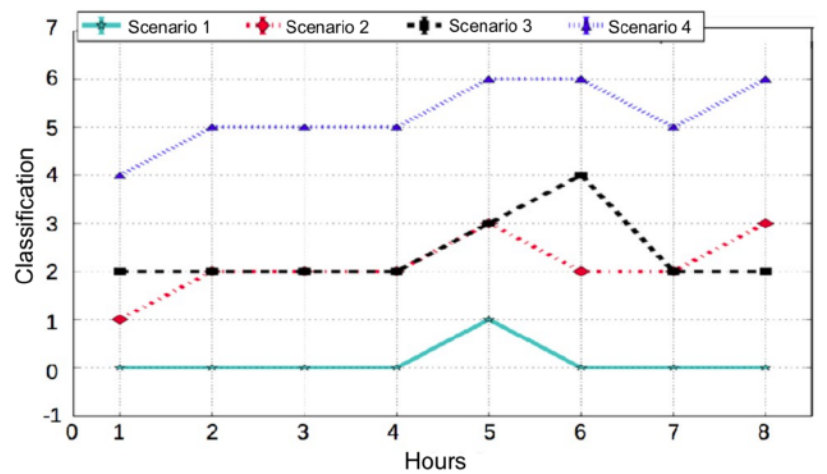

Figure 5: Scenario monitoring tests over an extended period.

It can be observed that from the evaluations carried out, both in terms of analyzing the sensitivity and quality of the data provided by the sensors and evaluating the system consistency in monitoring for a continuous period, that the system proved to be consistent and with high availability. This is according to their previously defined requirements and may provide evidence of the quality of the environment for the elderly to rest.

\section{CONCLUSION}

There are numerous methods, techniques and instruments that support monitoring for user health assessment, due to the opportunity for computer systems to react to the state and changing behavior of the environment or the individual, allowing computational applications to become aware of user context.

Therefore, this article investigated an infrastructure for Health "Smart" Homes that can detect and classify (automatically and without needing human intervention) aspects related to the environment for the elderly to rest. To this end, we used computational device sensors (brightness, noise and movement) that can monitor the environment in order to check the actual conditions or even possible causes of discomfort. This can provide an important aid in healthcare as issues related to rest can directly influence the behavior and well-being of the elderly population.

The results demonstrate that the proposed system allows continuous and non-intrusive monitoring of the quality of the environment which is coherent with reality. In addition, the system has been shown to function consistently by running for long periods. Moreover, the importance was observed of combining data from various sensors to provide a more assertive classification than using a single sensor regarding environmental monitoring for sleep quality, since data from a single sensor may not be sufficient to detect the quality of the environment for sleep.

However, technology is still a major challenge for the elderly and there is a need for further studies concerning a more assertive analysis of environmental conditions, not only for the elderly to rest, but for all daily living activities and even for the treatment of diseases, both in the hospital and at home. It is important to highlight that, based on the good results obtained, we identified the possibility of further studies which will be explored in order to improve the efficiency of the proposed system: (i) to study in depth the variability of environmental aspects to compose a quality metric; (ii) to explore machine learning algorithms to identify aspects of sleep; (iii) to explore the identification of emotional state on awakening as a sleep quality parameter; and (iv) to analyze if the individual's social interaction in the morning is related to the quality of their sleep.

The authors would like to thank the support from the São Paulo Research Foundation - FAPESP (processes 2018/25927-3 and 2019/22917-0) for funding part of this research project. We would also like to thank Professor Michael Biehl at the University of Groningen.

\section{REFERENCES}

[1] Colin DM, Gretchen AS, Ties B, Richard AW, Martin IT. Causes of international increases in older age life expectancy. The Lancet 2015; 385(9967): 540-548. https://doi.org/10.1016/S0140-6736(14)60569-9

[2] Mano L, Funes M, Volpato T, Neto J. Explorando tecnologias de loT no contexto de Health Smart Home: uma abordagem para detecÃßÃ£o de quedas em pessoas idosas. Journal of 
Ambient Intelligence and Humanized Computing 2016a; 2(1): 46-57. https://doi.org/10.26729/jadi.v2i1.1667

[3] Oliveira RN, Roth V, Henzen AF, Simao JM, Nohama P, Wille ECG. Notification oriented paradigm applied to ambient assisted living tool. IEEE Latin America Transactions 2018; 16(2): 647-653.

https://doi.org/10.1109/TLA.2018.8327425

[4] Mano LY, Faiçal BS, Nakamura LHV, Gomes PH, et al. Exploiting loT technologies for enhancing Health Smart Homes through patient identification and emotion recognition. Computer Communications 2016b; 89: 178-190. https://doi.org/10.1016/j.comcom.2016.03.010

[5] Mano LY, Faiçal BS, Vincius PG, Pessin G, et al. An intelligent and generic approach for detecting human emotions: a case study with facial expressions. Soft Computing 2019. https://doi.org/10.1007/s00500-019-04411-7

[6] Rialle V, Duchene F, Noury N, Bajolle L, Demongeot J. Health "smart" home: information technology for patients at home. Telemedicine Journal and E-Health 2002; 8(4): 395409.

https://doi.org/10.1089/15305620260507530

[7] Silva V, Ferreira V, Viana NS. Architecture for Integrating Healthcare Services to the Brazilian Digital TV System. IEEE Latin America Transactions 2015; 13(1): 241-249. https://doi.org/10.1109/TLA.2015.7040654

[8] Dohr A, Modre-Opsrian R, Drobics M, Hayn D, Schreier G. The internet of things for ambient assisted living. In: 2010 seventh international conference on information technology: new generations, 2010; pp. 804-809. https://doi.org/10.1109/ITNG.2010.104

[9] Kronbauer AH, da Luz HC, Campos J. Mobile Security Monitor: A Wearable Computing Platform to Detect and Notify Falls. IEEE Latin America Transactions 2018; 16(3): 957-965. https://doi.org/10.1109/TLA.2018.8358679

[10] Mano LY, Barros VA, Nunes LH, Sawada LO, et al. ENLACE: A Combination of Layer-Based Architecture and Wireless Communication for Emotion Monitoring in Healthcare. Mobile Information Systems 2019. https://doi.org/10.1155/2019/7329187

[11] Perera C, Zaslavsky A, Christen P, Georgakopoulos D. Context aware computing for the internet of things: A survey. IEEE communications surveys \& tutorials 2013; 16(1): 414454. https://doi.org/10.1109/SURV.2013.042313.00197
[12] Ancoli-Israel S, Poceta JS, Stepnowsky C, Martin J, Gehrman P. Identification and treatment of sleep problems in the elderly. Sleep medicine reviews 1997; 1(1): 3-17. https://doi.org/10.1016/S1087-0792(97)90002-2

[13] Ancoli-Israel S, Alessi C. Sleep and aging. The American Journal of Geriatric Psychiatry 2005; 13(5): 341-343. https://doi.org/10.1097/00019442-200505000-00001

[14] Ceolim MF, Diogo MJD, Cintra FA. Qualidade do sono de pessoas idosas atendidas no grupo de atençäo à saúde do idoso do Hospital das Clnicas da Universidade Estadual de Campinas. Nursing (Säo Paulo) 2001; 4(33): 25-9. https://doi.org/10.1109/SURV.2013.042313.00197

[15] Van Der Loos, HFM, Ullrich N, Kobayashi H. Development of sensate and robotic bed technologies for vital signs monitoring and sleep quality improvement. Autonomous Robots 2003; 15(1): 67-79.

https://doi.org/10.1023/A:1024444917917

[16] Chung WY, Oh SJ. Remote monitoring system with wireless sensors module for room environment. Sensors and Actuators B: Chemical 2006; 113(1): 64-70. https://doi.org/10.1016/j.snb.2005.02.023

[17] Carvalho ST, Erthal M, Mareli D, Sztajnberg A, et al. Monitoramento remoto de pacientes em ambiente domiciliar. XXVIII Simpósio Brasileiro de Redes de Computadores e Sistemas Distribudos-Salao de Ferramentas, Gramado, RS, Brasil, 2010; pp. 1005-1012.

[18] Togeiro SM, Smith AK. Diagnostics methods for sleep disorders. Revista brasileira de psiquiatria (Sao Paulo, Brazil: 1999) 2005; 27: 8-15. https://doi.org/10.1590/S1516-44462005000500003

[19] Campbell A, Choudhury T. From smart to cognitive phones. IEEE Pervasive Computing 2012; 11(3): 7-11. https://doi.org/10.1109/MPRV.2012.41

[20] Yao KW, Yu S, Cheng SP, Chen IJ. Relationships between personal, depression and social network factors and sleep quality in community-dwelling older adults. Journal of Nursing Research 2008; 16(2): 131-139. https://doi.org/10.1097/01.JNR.0000387298.37419.ff

[21] Horsten S, Reinke L, Absalom AR, Tulleken JE. Systematic review of the effects of intensive-care-unit noise on sleep of healthy subjects and the critically ill. British journal of anaesthesia 2018; 120(3): 443-452. https://doi.org/10.1016/j.bja.2017.09.006

\section{DOI: https://doi.org/10.31875/2409-9694.2020.07.1}

(c) 2020 Mano et al.; Zeal Press

This is an open access article licensed under the terms of the Creative Commons Attribution Non-Commercial License (http://creativecommons.org/licenses/by-nc/3.0/) which permits unrestricted, non-commercial use, distribution and reproduction in any medium, provided the work is properly cited. 